\section{Las reformas neoliberales del sector de la salud: déficit gerencial y alienación del recurso humano en América Latina}

\author{
Antonio Ugalde ${ }^{1}$ \\ y Nuria Homedes ${ }^{2}$
}

Palabras clave: reforma del sector salud, personal de salud, América Latina.

\footnotetext{
Departamento de Sociología, Universidad de Texas-Austin, EUA. La correspondencia debe dirigirse a Antonio Ugalde, 1808 Glencliff Dr., Austin, TX 78704, Estados Unidos de América. Dirección electrónica: augalde@mail.la.utexas. edu

2 Escuela de Salud Pública, Houston Health Science Center, Universidad de Texas, Houston, Texas, Estados Unidos.
}

El agotamiento del modelo neoliberal de reforma del sector de la salud es cada día más evidente (1-10). La reforma de salud neoliberal ha fracasado porque el modelo está fundamentado en principios económicos de rentabilidad y beneficio como los siguientes: el sector privado es más eficiente que el público y por ello la función del Estado no es producir bienes y servicios, sino regular; un mercado libre y competitivo genera bienes y servicios de mejor calidad que un mercado monopolístico; cuanto más cerca de la base se toman las decisiones, es más fácil controlar la corrupción, se atiende mejor a las necesidades locales y más satisfechos quedan los usuarios; y para incrementar la eficiencia y productividad de los servicios es necesario contar con una fuerza laboral flexible.

Los objetivos de las reformas están dirigidos a conseguir niveles aceptables de eficiencia, calidad y equidad en los servicios de atención médica y a aumentar la satisfacción de los usuarios (de aquí en adelante, objetivos de la reforma). Sin embargo, el propio Banco Mundial, principal propulsor del modelo neoliberal, ha empezado a reconocer que algunos de los postulados en los que se sustenta ese modelo no son los más adecuados en todos los contextos (11-12).

En este trabajo se discute cómo el sector privado puede ser menos eficiente que el público y cómo la flexibilidad laboral no produce los resultados esperados. También se analizan las fallas del proceso de reforma, especialmente las relacionadas con los recursos humanos, considerados por muchos observadores los más importantes y menos estudiados (13-16). Una vez presentados los problemas relacionados con los recursos humanos que se habían identificado antes de las reformas, se analizan las soluciones que estas han dado a los mismos. Para ello se consultaron fuentes secundarias - como informes, artículos, documentos y estudios etnográficos- y se realizaron entrevistas a profundidad a gestores del sector de la salud de Bolivia, Colombia, Costa Rica, Ecuador, El Salvador, México y República Dominicana. El análisis abarcó las tres últimas décadas.

\section{PROBLEMAS IDENTIFICADOS ANTES DE LA REFORMA}

Se estima que cerca de nueve millones de personas trabajan en el área de la salud en América La- 
tina y que este sector dedica casi $70 \%$ de sus recursos al pago de salarios. El uso intensivo de la mano de obra es importante para incrementar la eficiencia, la productividad y la calidad de los servicios. Es imposible alcanzar los objetivos de la reforma sin contar con la colaboración y el apoyo de los trabajadores.

Desde la década de 1970, varios autores demostraron la necesidad de introducir cambios en el área de recursos humanos (17-22).

\section{Problemas de recursos humanos}

Entre los problemas más importantes que afectaban - y que en muchos casos aún afectan-a este recurso se encuentran los siguientes:

Desproporción entre las diferentes categorías de profesionales y técnicos. No había suficientes técnicos para apoyar el trabajo de los profesionales, faltaban profesionales que no fuesen médicos y había un exceso de médicos especialistas. El desequilibrio entre el número de profesionales y de técnicos afecta directamente a la eficiencia y a la calidad de los servicios, ya que los primeros tienen que dedicar parte de su tiempo a llevar a cabo tareas que los técnicos pueden realizar mejor y a menor costo. El desequilibrio entre el número de profesionales que son médicos y que no lo son tiene un impacto directo en la calidad de los servicios y en la orientación curativa de la atención sanitaria, que puede resultar más costosa que las intervenciones preventivas, las acciones de promoción de salud y los tratamientos curativos no farmacológicos. El desequilibrio entre el número de médicos generales y de médicos especialistas conduce a prestaciones médicas más costosas y al uso innecesario de alta tecnología, con posibles consecuencias yatrógenas para el paciente, por lo que además de ser ineficiente puede afectar a la calidad de la atención. Si bien es cierto que los administradores, los economistas, los nutricionistas, los educadores y otros profesionales se incorporan paulatinamente a los equipos de salud, el proceso de toma de decisiones sigue bajo el control de los médicos, por lo que no ha habido cambios en el énfasis curativo de la actividad sanitaria, en detrimento de las acciones de salud pública. Según Bach (14), la carencia de personal en disciplinas tales como la atención primaria, la economía de la salud, la comunicación, la educación, la nutrición y la ingeniería sanitaria aún dificulta en gran medida que mejore la calidad y aumente la eficiencia en el sector de la salud.

Desproporción entre el número de profesionales de centros urbanos y rurales. Se observaba una concentración de personal en hospitales y centros de salud urbanos, al tiempo que no había suficiente personal médico y profesionales sanitarios en áreas rurales y urbanas marginales. Por una parte, era muy limitado el acceso de la población rural a todo tipo de servicios, tanto de salud pública como de atención curativa, lo que contribuía a una mayor inequidad. De otra parte, la concentración desproporcionada de recursos humanos en los hospitales también producía inequidad, porque la gran mayoría de los pobres tenían un acceso muy limitado a los mismos. Aunque esta realidad siempre ha existido y está ampliamente documentada, las reformas no han mejorado el acceso oportuno de las clases económicamente necesitadas a los servicios de salud. En Colombia, la reforma ha incrementado significativamente el aseguramiento de los más pobres, pero no su acceso a los servicios de salud, como se discute en otro artículo de este mismo número de la Revista Panamericana de Salud Pública (23).

El pluriempleo médico. Esta es una realidad también conocida desde hace muchos años y una de las causas del absentismo laboral en las instituciones públicas y de la baja productividad de los médicos en ese sector. Además, es una fuente de conflictos de intereses para los médicos que remiten a sus pacientes en el sector público a los servicios privados. Según Brito (24), el pluriempleo provocado por la reforma ha aumentado el estrés y el descontento entre los médicos.

Falta de control gerencial de los recursos humanos. Los gerentes se encontraban en la difícil tarea de administrar reglamentos que habían sido elaborados por otras instituciones (25). Elementos gerenciales básicos, tales como la definición del salario, la promoción, el despido, la carga laboral, los horarios de trabajo, la ubicación y la transferencia del personal, quedaban fuera del control de los administradores de salud. Unas veces las decisiones eran el resultado de negociaciones políticas entre el Gobierno y los sindicatos, y otras veces provenían de otros ministerios, como los de finanzas, educación, trabajo o servicio civil. En aquellos casos en los que el sector de la salud o sus instituciones podía contratar personal, tanto la selección de la persona como los criterios de promoción con frecuencia no respondían a necesidades técnicas, a la capacidad o a la experiencia profesional de los candidatos, sino a favoritismos, nepotismos y dictados políticos. Los ministerios de salud y las entidades encargadas de la seguridad social no controlaban la formación del personal profesional y técnico ni podían decidir el número de profesionales y técnicos de cada categoría que necesitaban. Un país podía tener al mismo tiempo un exceso de médicos que presionaban para 
conseguir plazas y una carencia de personal de enfermería. Todo ello limitaba la capacidad gerencial. La solución de estos problemas es compleja y requiere la participación de varios ministerios, la existencia de reglamentos que se cumplan y sistemas judiciales que fallen contra la contratación y los despidos injustos. Es también necesario emprender difíciles negociaciones políticas entre el Gobierno, los sindicatos y las asociaciones profesionales. No se han percibido mejoras en este sentido como consecuencia de las reformas.

Poca capacidad gerencial. Esto se observaba especialmente en los hospitales que consumían la mayor parte de los recursos destinados a la salud. Además del absentismo ya mencionado, se observaba con frecuencia una falta de control de la calidad y de los recursos básicos debido a carencia de presupuesto, incompetencia gerencial y robo. A pesar del interés demostrado por la reforma en mejorar la gerencia hospitalaria y en optimizar el uso de recursos, la eficiencia y la calidad de los servicios de un gran número de hospitales siguen siendo muy bajas $(26,27)$.

Débil regulación de la práctica profesional. La regulación deficiente hacía que se evadiera con facilidad el cumplimiento de las leyes. Su impacto en la calidad de los servicios y el peligro que supone para la salud de la población son obvios. Poco han hecho las reformas por resolver este problema. Por ejemplo, en muchos países es obligatorio que las farmacias estén atendidas por lo menos por un farmacéutico profesional; sin embargo, el incumplimiento de esta regulación está documentado en la mayoría de los países de la Región.

Deficiente comunicación entre los proveedores de salud y los usuarios. Las diferencias de clase social, la asimetría de conocimientos y, en regiones multiculturales, el desconocimiento de las culturas amerindias y de sus idiomas por parte de los médicos y otros profesionales dificultan la comunicación con los pacientes. Sin una buena comunicación entre los proveedores de salud y los pacientes, la calidad de la atención se ve afectada. Las reformas no han contribuido a la solución de este problema.

\section{RESPUESTAS DE LAS REFORMAS A LOS PROBLEMAS DE RECURSOS HUMANOS}

Frente a estas necesidades ya reconocidas y las nuevas necesidades generadas por los cambios introducidos por la reforma (descentralización, autonomía hospitalaria, privatización de servicios, entre otros), sus defensores ofrecieron diversos programas para mejorar la gestión de los recursos humanos.

\section{Programas de la reforma en recursos humanos}

Entrenamiento gerencial. Este entrenamiento estaba dirigido a futuros administradores de servicios descentralizados, así como a administradores de hospitales, y abarcaba el uso de paquetes informáticos y de sistemas de información que facilitaran la aplicación de los nuevos métodos de financiación y facturación $(28,29)$. Una evaluación realizada por la Organización Panamericana de la Salud (OPS) a 15 programas de entrenamiento concluyó que estos no habían mejorado la capacidad gerencial (30). Bach (14) indica que las unidades de recursos humanos no tenían la capacidad técnica para poner en práctica las tareas que les impusieron los planificadores de las reformas, entre ellas el entrenamiento de personal. Un estudio realizado en el estado de Baja California Sur, México, confirmó la falta de preparación del personal para las reformas (31), ya demostrada en otros estados de México $^{3}$. Evaluaciones realizadas en Brasil en 2003 llegaron a la conclusión de que la calidad de los servicios hospitalarios no había mejorado y posiblemente era peor que antes de la reforma (27). Una evaluación realizada en Guatemala llegó a la misma conclusión, a pesar de haber recibido un préstamo del Banco Interamericano para el Desarrollo con fondos destinados a este objetivo (26). En Colombia, a partir de la reforma, los hospitales han empezado a sufrir una crisis administrativa desconocida en la historia moderna de ese país y muchos de ellos están en quiebra (32).

El Programa Flagship. Este es un programa disenado por el Banco Mundial para promover la reforma neoliberal entre los altos ejecutivos de los servicios de salud mediante un equipo de docentes seleccionados por esa entidad para cada región del mundo. Al principio, la selección de los participantes en este programa era muy cuidadosa y solía limitarse a ejecutivos de los países donde se estaban diseñando e aplicando reformas, para lo cual se utilizaban los préstamos otorgados para la implantación de las reformas.

Adiestramiento en la preparación y administración de contratos. Las reformas neoliberales requieren de una mano de obra flexible que permita despedir a un empleado cuando el empleador lo considere necesario. Para evitar despidos arbitrarios se requiere establecer contratos que especifiquen las razones justificadas de despido. La priva-

\footnotetext{
Homedes N, Ugalde A, eds. Transforming the state: the decentralization of health services in Mexico; 2005. (Manuscrito sin publicar, disponible a quien lo solicite).
} 
tización de servicios promovida por las reformas también exige que se establezcan contratos entre las instituciones de financiamiento y los proveedores de los servicios, lo que requiere capacidad para evaluar los contratos de los seguros privados médicos y conocer el sistema de pago según el desempeño. El conocimiento sobre estas áreas gerenciales complejas era muy limitado antes de las reformas (33) y siguió siendo deficiente a pesar del adiestramiento, que con frecuencia comprendía costosas asesorías por parte de firmas extranjeras (comunicación personal: entrevistas con dos consultores extranjeros del Instituto Mexicano de Seguridad Social [IMSS] el 6 de marzo de 2002 y entrevistas con tres ejecutivos del nivel federal del IMSS los días 28 y 29 de septiembre de 2003).

\section{La flexibilización de la mano de obra}

El neoliberalismo ha promovido la flexibilización de la fuerza laboral a fin de reducir los costos e incrementar la eficiencia. Para conseguir el primer objetivo es necesario limitar algunos derechos y beneficios conquistados por los trabajadores a lo largo de años de luchas laborales, como la seguridad en el trabajo, las jubilaciones y los salarios justos. Además, se debe establecer el derecho al despido inmediato según la necesidad de los gestores e instituir sistemas de pago por desempeño y los llamados "contratos basura", es decir, contratos temporales con remuneraciones muy bajas y sin derecho a la jubilación ni a otras prestaciones.

La defensa de los derechos de los trabajadores mediante la sindicalización y la permanencia en el trabajo que otorga la administración pública crean monopolios; sin embargo, según los conceptos neoliberales, los monopolios impiden la eficiencia. Para incrementar la eficiencia es necesario que el gerente tenga el control absoluto de la organización del trabajo. La flexibilidad permite a los gerentes imponer libremente condiciones laborales, por ejemplo, la carga laboral, los horarios, las nuevas actividades a realizar y cambios del lugar de trabajo. Si el empleado no las acepta, el gerente puede despedirlo libremente. Según la doctrina neoliberal, el temor de los trabajadores a perder su empleo aumenta la productividad y mejora la calidad.

Las evaluaciones realizadas a las reformas indican que la flexibilidad de la mano de obra raramente consigue los objetivos buscados y que, por el contrario, puede empeorar la situación. Un estudio del personal de enfermería realizado en Argentina, Colombia, México y Brasil (34) demostró el impacto negativo de la flexibilización laboral en la calidad. Según ese estudio, las reformas aumentaban el estrés y la insatisfacción de los trabajadores, su inse- guridad por los contratos flexibles de trabajo, la preocupación por posibles juicios por negligencia profesional, la migración laboral entre diferentes instituciones y añadían nuevas labores burocráticas para las cuales el personal de enfermería no estaba entrenado. Específicamente, los enfermeros y enfermeras colombianos mencionaron que tenían que realizar más actividades en menos tiempo y con menos personal de apoyo, y se quejaban de tener menos tiempo para atender a los pacientes que antes de la reforma. Una enfermera comentó: "Los pacientes pensarán que nos importan muy poco, porque realmente no tenemos tiempo para cuidarlos y en verdad no sabemos lo que les está pasando" (34). Según otro estudio, los enfermeros y enfermeras que habían recibido un entrenamiento especial para adaptarse a las reformas tenían percepciones más negativas sobre el cuidado de los pacientes que los que no habían recibido ese entrenamiento (13). Otros estudios $(28,35)$ coinciden en que el énfasis en la productividad puede disminuir la dedicación a los pacientes y hacer que se dejen de realizar actividades importantes que requieren tiempo adicional, como establecer una buena comunicación entre el médico y el paciente o la educación de salud ${ }^{4}$.

Las presiones para aumentar el ahorro llevan a los gerentes a reducir los gastos de mantenimiento y los insumos. Sin los recursos materiales adecuados, la calidad del trabajo de los proveedores se pone en entredicho (36), lo cual explica el temor expresado por el personal de enfermería colombiano y los médicos mexicanos a enfrentarse a juicios por mala práctica ${ }^{5}$. Por otra parte, cuando el hospital compra equipos de alta tecnología se ejercen presiones sobre los médicos para que los utilicen, sea o no necesario, especialmente cuando una empresa administradora de seguros de salud paga los gastos. Esta demanda innecesaria influye negativamente en la calidad de los servicios de salud, ya sea por la falta de una adecuada infraestructura o por sus efectos yatrógenos.

La mayoría de los trabajadores del sector de la salud consideran que las reformas neoliberales van en contra de sus intereses. Los empleados públicos saben que, dada la realidad política de América Latina, es necesario contar con garantías de permanencia laboral para evitar despidos arbitrarios cada vez que cambia el Gobierno. Para proteger los derechos adquiridos, los sindicatos se han opuesto firmemente a las reformas de salud en Bolivia, Ecuador, El Salvador, México, Nicaragua, Perú y Venezuela

\footnotetext{
4 Dussault G. Human resources development: the challenge of health sector reform; 1999. (Manuscrito inédito, disponible a quien lo solicite).

5 Abrantes Pego R. La lenta y difícil institucionalización de la reforma del sector salud en Sonora: 1982 a 2000; 2004. (Manuscrito inédito, disponible a quien lo solicite).
} 
(37), y han conseguido evitar la privatización de los servicios de salud en Argentina, El Salvador y México $(1,38,39)$. Los temores de los sindicatos se han confirmado en años recientes, ya que disminuyó el número de trabajadores sindicalizados (13) y aumentó notablemente el número de trabajadores contratados temporalmente sin los beneficios laborales que ofrece la administración pública en Argentina (34), Colombia (40), Ecuador (35), El Salvador (24), México ${ }^{3}$, Panamá (24) y Perú (35).

Previendo la oposición de los trabajadores, las reformas se han diseñado secretamente en los ministerios de salud, en oficinas paralelas e independientes de las de planificación ${ }^{5}(25,37)$, y su aplicación se ha impuesto por mandato, "desde arriba", sin la participación de los gerentes provinciales y sin consultar a los trabajadores. El sigilo del proceso ha provocado rumores, confusión e incluso hostilidad $(8,24,37,38,41)$.

Las reformas no han conseguido aumentar la eficiencia. En un estudio sobre las reformas de administración civil en 15 países, el Banco Mundial reconoció que los préstamos concedidos para aumentar la eficiencia no consiguieron "mayor eficiencia/ productividad en la administración civil" en ninguno de los países, según citaron Bennet y Franco en 1999 (42). El énfasis exagerado en la compensación monetaria puede desplazar otros valores que son fuente importante de motivación para mejorar la eficiencia y la calidad, como el placer de hacer las cosas bien o de ayudar a los pacientes. Incluso la lealtad a la institución puede desaparecer cuando la compensación económica es la única fuente de satisfacción, ya que los trabajadores estarán siempre en búsqueda de mejores oportunidades en otras instituciones. En Brasil, el establecimiento de un sistema de compensación basado en la productividad logró incrementarla en un inicio, pero no pudo mantenerla durante mucho tiempo y provocó la competencia entre trabajadores que debían colaborar entre sí (43).

La crítica a la flexibilidad laboral no es un respaldo a la inflexibilidad de los sindicatos. Hay antecedentes de que los sindicatos se han opuesto a modificaciones necesarias de los horarios, a incrementos razonables de la carga laboral, a cambios en la organización del trabajo que respondan a las nuevas tecnologías o de que han exigido privilegios costosos que la gran mayoría de la población no disfruta. Algunos sindicatos han estado controlados por cúpulas corruptas que se perpetúan en el poder sin mostrar interés en facilitar cambios y reformas necesarios para mejorar la gerencia y la calidad de los servicios y aumentar la satisfacción de los usuarios. La solución de estos problemas debe ser política y no puede contradecir derechos fundamentales universalmente reconocidos, como el derecho de los trabajadores a organizarse.

\section{RENOVAR SÍ, PERO ¿CÓMO?}

La crítica a las reformas neoliberales no niega la necesidad de modificar e innovar elementos relacionados con los recursos humanos para conseguir los mismos objetivos que plantean las reformas. Este análisis cuestiona los principios en los que está sustentada esa reforma y el proceso seguido tanto para su diseño como para su puesta en marcha. Los cambios hay que buscarlos dentro de los principios y los valores que históricamente propulsaron la organización del sector de la salud en América Latina, es decir, en la solidaridad más que en la competitividad y en la ayuda más que en el beneficio. Estos principios siguen siendo válidos y viables.

Todas las sociedades necesitan vivir en un estado permanente de cambio para superar ineficiencias, incorporar innovaciones tecnológicas, solucionar problemas que se han resistido al cambio y resolver los nuevos conflictos que surgen. Cuando las instituciones públicas dejan de ofrecer los bienes o los servicios esperados por los ciudadanos - por inercia, por satisfacer los intereses económicos o políticos de algunos grupos, o por la falta de competencia de sus dirigentes que hace que se pierda la dinámica de cambio- surgen las crisis sociales y políticas. Por ello es necesario realizar un examen continuo del quehacer institucional del sector público.

Excepto en situaciones en que las condiciones del país exigen cambios revolucionarios, los objetivos de las reformas se pueden conseguir mejor $-\mathrm{y}$ probablemente a menor costo- mediante cambios puntuales, progresivos y con la aplicación de correcciones específicas a las políticas ya existentes, en lugar de reformas que intentan cambiar los valores, especialmente si no existen los requisitos legales o políticos necesarios. Antes de desmontar las instituciones construidas a lo largo de los años, que a pesar de sus muchos defectos han acumulado una valiosa experiencia, hay que asegurarse de que son obsoletas e incorregibles.

En la historia contemporánea de América Latina hay muchos ejemplos de valiosas innovaciones llevadas a cabo por el sector público. Algunas consiguieron resultados muy positivos; otras quedaron a medio camino, pero hubieran conseguido los efectos deseados si una reforma basada en principios menos materialistas las hubiera apoyado. Otras, por diversas razones, fracasaron.

Además de la transformación radical del sector de la salud en Cuba, resultado de su revolución política, se puede mencionar la fusión de los hospitales y clínicas del Ministerio de Salud de Costa Rica con los de la Caja Costarricense de Seguridad Social. Este cambio ha conseguido, a pesar de contar con escasos recursos, uno de los sistemas de salud más equitativos y mejor organizados de la 
Región, aunque no esté exento de problemas. A pesar de ello, se observa interés y se ejercen presiones para sustituirlo por uno de corte neoliberal.

En México, uno de los mejores programas de extensión de la cobertura sanitaria en zonas rurales marginadas fue el patrocinado por el IMSS y la Coordinación General del Plan Nacional de Zonas Deprimidas y Grupos Marginales (COPLAMAR) denominado IMSS-COPLAMAR (44). El Banco Mundial, sin hacer distinciones entre los diferentes programas que ofrecía COPLAMAR, lo calificó como "ejemplo clarísimo" (glaring example) de programa insostenible (45) e hizo todo lo posible por terminarlo. En su lugar apoyó un programa neoliberal denominado SEDESOL, que el ex presidente Salinas de Gortari utilizó para promover el nepotismo político y que terminó al finalizar su período presidencial. Debido a la descentralización apoyada por el Banco Mundial, el programa IMSS-COPLAMAR transfirió un grupo de hospitales a los servicios estatales descentralizados, lo que redujo notablemente la calidad de los servicios.

Entre los programas que han tenido un éxito innegable se encuentra el de la producción pública de medicamentos en Brasil, a pesar de que el Banco Mundial siempre ha sostenido que la producción pública de medicamentos es ineficiente (46). Si el gobierno brasileño hubiera seguido las recomendaciones del Banco Mundial, hubieran muerto miles de brasileños que sufren de infección por VIH o sida. El Hospital del Niño, en Panamá, y el Hospital sin Paredes, en Costa Rica, ambos públicos, son ejemplos de participación comunitaria el primero y de servicio y dedicación profesional a la comunidad rural dispersa, el segundo. Este último dejó de funcionar por falta de apoyo.

Otro programa innovador que se ha debilitado es el Seguro Campesino de Ecuador. Caracterizado por una gran participación popular y por buscar una mayor equidad, este programa podría haber solucionado las necesidades de salud de las comunidades más pobres del país si hubiera contado con mayor apoyo. Contra las protestas de los campesinos, la reforma neoliberal ha intentado reducir el Seguro Campesino a la entrega de un paquete mínimo de servicios (47).

No se necesitan más ejemplos para demostrar la capacidad de solución que tiene el sector público en América Latina. Propiciar y apoyar esa capacidad ofrece más posibilidades de conseguir buenos resultados a menor costo que las reformas neoliberales, según lo visto hasta el momento.

\section{CONCLUSIONES}

Después de una inversión de muchos millones de dólares en diseñar y poner en marcha refor- mas neoliberales, los sistemas de salud de América Latina no son más equitativos, más eficientes ni de mejor calidad que antes de las reformas. Se hubieran obtenido mejores resultados si en lugar de imponer estrategias que no están basadas en la evidencia y que responden a una agenda ideológica, los promotores de las reformas se hubieran preocupado por evaluar y fortalecer algunas de las iniciativas generadas por los propios países.

Muchas de las soluciones a los problemas que plantean los recursos humanos requieren la colaboración de diferentes ministerios e instituciones, y el Banco Mundial tiene la posibilidad de apoyar ese proceso de coordinación. Los sistemas de salud cuyos profesionales no estén motivados para trabajar en equipo a favor de la salud de la población están condenados al fracaso. Los países tendrán que invertir tiempo y recursos en recuperar el compromiso de los profesionales con la población y con el sistema y deberán abandonar su orientación mercantilista.

\section{SYNOPSIS}

\section{Neoliberal health sector reforms in Latin America: unprepared managers and unhappy workers}

This work analyzes the neoliberal health sector reforms that have taken place in Latin America, the preparation of health care workers for the reforms, the reforms' impacts on the workers, and the consequences that the reforms have had on efficiency and quality in the health sector. The piece also looks at the process of formulating and implementing the reforms. The piece utilizes secondary sources and in-depth interviews with health sector managers in Bolivia, Colombia, Costa Rica, the Dominican Republic, Ecuador, El Salvador, and Mexico. Neoliberal reforms have not solved the human resources problems that health sector evaluations and academic studies had identified as the leading causes of health system inefficiency and low-quality services that existed before the reforms. The reforms worsened the situation by putting new pressures on health personnel, in terms of both the lack of necessary training to face the challenges that came with the reforms and efforts to take away from workers the rights and benefits that they had gained during years of struggles by unions, and to replace them with temporary contracts, reduced job security, and lower benefits. The secrecy with which the reforms were developed and applied made workers even more unified. In response, unions opposed the reforms, and in some countries they were able to delay the reforms. The neoliberal reforms have not improved the efficiency or quality of health systems in Latin America despite the resources that have been invested. Nor have the neoliberal reforms supported specific changes that have been applied in the public sector and that have demonstrated their ability to solve important health problems. These specific changes have produced better results than the neoliberal reforms, and at a lower cost.

Key words: health sector reform, health personnel, Latin America. 


\section{REFERENCIAS}

1. Lloyd-Sherlock P. Health sector reform in Argentina: a cautionary tale. Soc Sci Med. 2005;60(8):1893-1903

2. Verdugo JC. Los riesgos de la reforma del sector salud y el papel de la sociedad civil en Guatemala: entre una economía de guerra y una economía de mercado. [Presentación en taller]. En: Seminario Taller sobre Desarrollo Humano y Salud en la Nueva Economía Global: Experiencias, Oportunidades y Riesgos en las Américas. University of Texas, Medical Branch, Galveston TX, marzo de 1999.

3. Homedes N, Ugalde A. Why neoliberal reforms have failed in Latin America. Health Policy. 2005;71(1):83-96.

4. Plaza B, Barona AB, Hearst N. Manager competition for the poor or poorly managed competition? Lessons from the Colombian health reform experience. Health Policy Plan. 2001;12(suppl 2): 44-51.

5. Barrientos A. Health policy in Chile: the return of the public sector? Bull Lat Am Res. 2002;21(3):442-59.

6. Sapelli C. Risk segmentation and equity in the Chilean mandatory health insurance system. Soc Sci Med. 2004;58:259-65.

7. Hernández M. Reforma sanitaria, equidad y derecho a la salud en Colombia. Cad Saude Publica. 2002;18(4):991-1001.

8. Salinas H, Lenz R. Las no reformas de salud en Latinoamérica, razones que explican su fracaso. Santiago, Chile: Andros; 1999.

9. Evans T, Whitehead M, Diderichsen F Bhuiya A. Wirth M. Challenging inequities in health. From ethics to action. Oxford: Oxford University Press; 2001.

10. Birn A. Federalist flirtations: the politics and execution of health services decentralization for the uninsured populations in México, 1985-1995. J Public Health Policy. 1999;20(1):81-108.

11. World Bank. Colombia. Towards increased efficiency and equity in the health sector. Can decentralization help? Washington, D.C.: World Bank; 1994. (Sector Report No. 11933-CO).

12. World Bank. World Development Report 2004. Making services work for the poor. Oxford: Oxford University Press; 2004.

13. Rigoli F, Dussault G. The interface between health sector reform and human resources in health. Hum Resour Health. 2003;1:9. Hallado en: http:www. human-resources-health.com/content/ 1/1/9. Acceso el 14 de febrero de 2005.

14. Bach S. Human resources and new approaches to public sector management. [Presentación en taller]. En: Global Health Workforce Strategy, Annecy (Francia), 9-12 de diciembre, 2000.

15. Brito P, Galin P, Novick M. Labour relations, employment conditions and participation in the health sector. [Presentación en taller]. En: Global Health Workforce Strategy, Annecy (Francia), 9-12 de diciembre de 2000.
16. Homedes N, Ugalde A. Human resources: the Cinderella of health sector reform in Latin America. Hum Resour Health. 2005;3:1. Hallado en: http:// www.human-resources-health.com/ content $/ 3 / 1 / 1$. Acceso el 20 de febrero de 2005.

17. United Nations Agency for International Development. Bolivia health sector assessment. La Paz: USAID; 1975.

18. McGreevey W. Brazilian health care financing and health policy: an international perspective. Washington, D.C.: World Bank; 1982.

19. United Nations Agency for International Development. Un análisis del sector colombiano de salud pública. Santa Fe de Bogotá: USAID; 1972

20. LeBow R, McCarthy D, Cross P. Project evaluation summary specific to the rural health delivery system (SBS) in the Dominican Republic. Health Sector Loans I and II. Evaluation and recommendations. Boston: Management Sciences for Health; 1983.

21. United Nations Agency for International Development. Health sector assessment: Guatemala. Guatemala: USAID; 1977.

22. United Nations Agency for International Development. Health sector assessment for Nicaragua. Managua: USAID; 1976.

23. Homedes N, Ugalde A. Las reformas de salud neoliberales en América Latina: una visión crítica a través de dos estudios de caso. Rev Panam Salud Publica. 2005;17(3):210-20

24. Brito P. Impacto de las reformas del sector salud sobre los recursos humanos y la gestión laboral. Rev Panam Salud Publica. 2000;8(1/2):43-54.

25. Biscoe G. Human resources: the political and policy context. [Presentación en taller]. En: The Global Workforce Strategy Group. Geneva: WHO; 2001

26. Sánchez Viesca A. La práctica de la reforma del sector salud en Guatemala: lecciones para el futuro. Guatemala: Centro para el Desarrollo Estratégico en Salud; 2000. (Publicación Científica No.1)

27. La Forgia GM. In search of excellence. Strengthening hospital performance in Brazil. Concept paper. Washington, D.C.: World Bank; 2003.

28. Egger D, Lipson D, Adams O. Issues in health services delivery: human resources for health. Geneva: World Health Organization; 2000. (WHO/EIP/ OSD/00.2)

29. Pan American Health Organization. Human resources: a critical factor in health sector reform. [Presentación]. En: Reunión Regional de la OPS, San José, Costa Rica, 3-5 diciembre, 1997.

30. Organización Panamericana de la Salud. Situación de los componentes educacionales en proyectos relacionados con los procesos de reforma del sector salud. Programa de desarrollo de los re- cursos humanos. Washington, D.C.: OPS; 2001.

31. Olvera Santana L. Análisis de la implementación de la descentralización de los servicios de salud en el Estado de Baja California Sur 1996-2000 [Tesis de maestría]. Cuernavaca, México: Instituto Nacional de Salud Pública; 2002.

32. Tono T. La crisis de los hospitales de Colombia. [Presentación]. En: The Innovations in Health Financing, México D.F., 20-21 de abril de 2004. Hallado en http:/ / conferencias.salud.gob.mx/ ingles/pres_22_de_abril_ingles.html. Acceso el 14 de febrero de 2005.

33. Adams O, Hicks V. Pay and non-pay incentives, performance and motivation. [Presentación en taller]. En: Global Health Workforce Strategy Group, Annecy, Francia, 9-12 de diciembre de 2000.

34. Guevara EB, Mendias EL. A comparative analysis of the changes in nursing practices related to health sector reform in five countries of the Americas. Rev Panam Salud Publica. 2002;12:347-53.

35. International Labor Organization. Terms of employment and working conditions in health sector reforms. Report for discussion at the Joint Meeting on Terms of Employment and Working Conditions on Health Sector Reforms. Geneva: ILO; 1998.

36. Buchan J, Thompson M, O'May F. Health workforce incentive and remunerationa research review. Geneva: World Health Organization; 2000. (WHO/EIP/ OSD $/ 00$ ).

37. Maceira D, Murillo V. Social sector reform in Latin America. Washington, D.C.: Inter-American Development Bank; 2001. (IDB, Research Department, Working paper 456).

38. Homedes N, Paz C, Solas O, SelvaSutter E, Ugalde A. Health reform in El Salvador: theory and practice. En: Peter Lloyd-Sherlock, ed. Health care reform and poverty in Latin America. London: Institute of Latin American Studies, University of London; 2000.

39. Acosta Córdova C, Correa G. Negocia el gobierno a espaldas del Congreso un crédito de 700 millones de dólares para financiar la "reforma" de la seguridad social. Proceso (México). 1998;29:1117.

40. Schlette S. Public service reform and their impact on health sector personnel in Colombia. Geneva: ILO, World Health Organization; 1998.

41. Public Service International. The IDB, the World Bank, labor rights and health care privatization in the Americas. Washington, D.C.: PSI; 2001.

42. Bennet S, Franco LM. Public sector health worker motivation and health sector reform: a conceptual framework. Bethesda, MD: ABT Associates, Partnerships for Health Care Reform; 1999.

43. Leal Cherchiglia M, Nicolau Girardi S, de Castro Vieira R, Bibiani de Aguilar 
Marques R, Mendes Werneck da Rocha P, Cimino Pereira LA. Remuneración y productividad: el caso de la Fundación Hospitalaria del Estado de Minas Gerais, Brasil, 1992-1995. Rev Panam Salud Publica. 1998;4(2):112-20.

44. Gershberg AI. Decentralization and recentralization: lessons from the social sectors in Mexico and Nicaragua. Washing- ton, D.C.: Inter-American Development Bank; 1998. (Final Report RE2/SO2).

45. World Bank. Mexico: decentralization and regional development project for the disadvantage states. Washington, D.C.: World Bank; 1991. (Report No. 8786-ME).

46. World Bank. World Development Report 1993. Investing in Health. Oxford: Oxford University Press; 1993.
47. Cárdenas M. El Seguro Social Campesino en Ecuador. [Presentación]. En: Gestionando la mejora de la salud: la función de rectoría de los ministerios de salud. Foro de Europa y las Américas sobre Reforma del Sector Salud, Málaga, España, 24-27 de febrero, 2002.

\section{"Medicine and Health 2005"}

The 2005 version of "Medicine and Health," a guide to 2,500 of the world's best training courses, graduate and postgraduate programs, conferences and other continuing education opportunities in the health sciences, is now available. The information, which is presented in an organized, coherent manner, is essential to professionals in health-related fields-physicians, managers of health care programs, health policy-makers, members of non-profit organizations involved in international development cooperation and humanitarian aid, etc. - who wish to further their skills and knowledge through additional training. The learning opportunities described in the book are offered in 700 prestigious academic institutions or organizations in 45 countries throughout the world. In addition to details on course

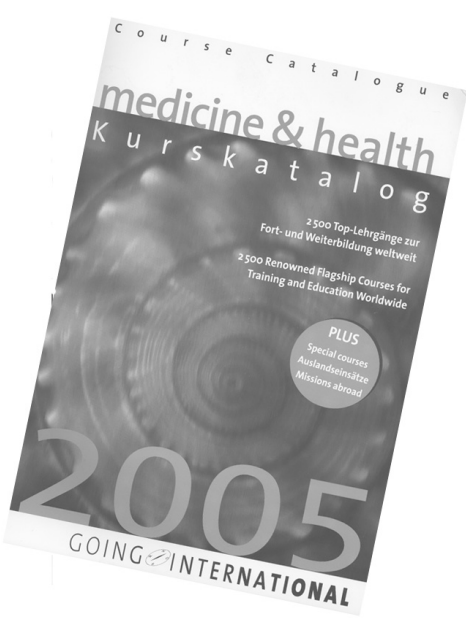
contents, requirements and qualifications, and the registration process, the book provides 150 links to relevant websites and 50 international journals, as well as interesting articles by wellknown scientists.

For orders and further information, please contact:

GOING INTERNATIONAL; Information Services G.Polak KEG; Gerhard Polak, MD; Fasangasse 28/27; 1030 Wien; Tel. 01/798 25 27-15; E-Mail: media@goinginternational.org; http://www.goinginternational.org 